\title{
Correction: Allogeneic transplantation in elderly patients $\geq 65$ years with non-Hodgkin lymphoma: a time-trend analysis
}

Nirav N. Shah, Kwang Woo Ahn, Carlos Litovich, Anna Sureda, Mohamed A. Kharfan-Dabaja (D, Farrukh T. Awan, Siddhartha Ganguly, Usama Gergis, David Inwards, Reem Karmali, Alexsandr Lazaryan, Lazaros Lekakis, Pashna Munshi, Sunita Nathan, Ayman A. Saad, Melhem Solh, Amir Steinberg, Ravi Vij, William A. Wood, Timothy S. Fenske, Sonali Smith and Mehdi Hamadani

\section{Correction to: Blood Cancer Journal https://doi.org/10.1038/s41408-019-0261-1 published online 3 December 2019}

The original version of the article contained incorrect acknowledgements. The correct ones are:

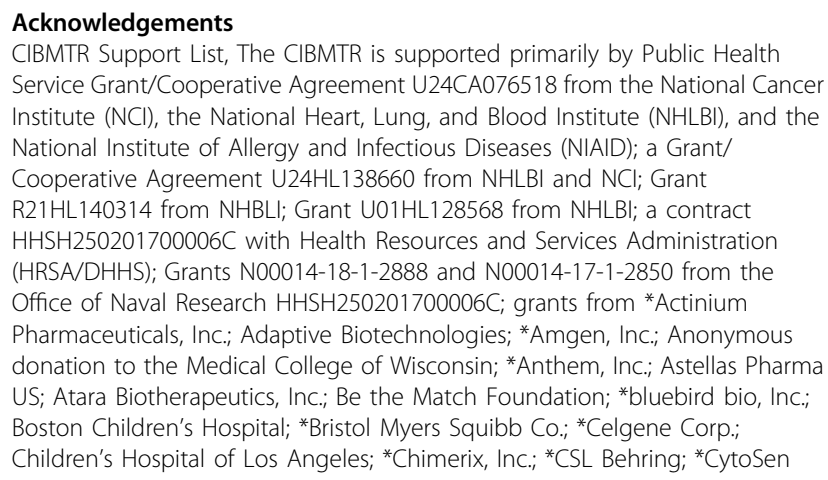
Service Grant/Cooperative Agreement U24CA076518 from the National Cancer Institute (NCI), the National Heart, Lung, and Blood Institute (NHLBI), and the National Institute of Allergy and Infectious Diseases (NIAID); a Grant/ Cooperative Agreement U24HL138660 from NHLBI and NCl; Grant R21HL140314 from NHBLI; Grant U01HL1 28568 from NHLBl; a contract HHSH250201700006C with Health Resources and Services Administration (HRSA/DHHS); Grants N00014-18-1-2888 and N00014-17-1-2850 from the Office of Naval Research HHSH250201700006C; grants from *Actinium Pharmaceuticals, Inc.; Adaptive Biotechnologies; *Amgen, Inc.; Anonymous donation to the Medical College of Wisconsin; *Anthem, Inc.; Astellas Pharma US; Atara Biotherapeutics, Inc.; Be the Match Foundation; *bluebird bio, Inc.; Boston Children's Hospital; *Bristol Myers Squibb Co.; ${ }^{*}$ Celgene Corp.;

Children's Hospital of Los Angeles; ${ }^{*}$ Chimerix, Inc:; ${ }^{*}$ CSL Behring; *CytoSen

Therapeutics, Inc.; Dana Farber Cancer Institute; *Daiichi Sankyo Co., Ltd.; Fred Hutchinson Cancer Research Center; * Gamida-Cell, Ltd.; Gilead Sciences, Inc.; *GlaxoSmithKline (GSK); HistoGenetics, Inc.; Immucor; Incyte Corporation; Janssen Biotech, Inc:; *Janssen Pharmaceuticals, Inc.; Janssen Scientific Affairs, LLC; *Jazz Pharmaceuticals, Inc.; Karius, Inc.; Karyopharm Therapeutics, Inc.; *Kite, a Gilead Company; *Magenta Therapeutics; Medac GmbH; The Medical College of Wisconsin; Mediware; Merck \& Company, Inc.; ${ }^{*}$ Mesoblast; MesoScale Diagnostics, Inc.; Millennium, the Takeda Oncology Co.; *Miltenyi Biotec, Inc.; Mundipharma EDO; National Marrow Donor Program; Novartis Oncology; Novartis Pharmaceuticals Corporation; *Omeros Corporation; *Oncoimmune, Inc.; PCORI; *Pfizer, Inc:; *Phamacyclics, LLC; PIRCHE AG; *Regeneron Pharmaceuticals, Inc.; REGiMMUNE Corp.; *Sanofi Genzyme; *Seattle Genetics; *Shire; Sobi, Inc.; Spectrum Pharmaceuticals, Inc.; St. Baldrick's Foundation; Swedish Orphan Biovitrum, Inc.; *Takeda Oncology; University of Minnesota; University of Pittsburgh; University of Texas-MD Anderson; University of Wisconsin-Madison and Viracor Eurofins. The views expressed in this article do not reflect the official policy or position of the National Institute of Health, the Department of the Navy, the Department of Defense, Health Resources and Services Administration (HRSA), or any other agency of the U.S Government. * Corporate members: Kristina Jacobs for administrative support.

The original article has been updated.

Published online: 29 April 2021 